\title{
COVID-19 pandemic: a new cause of unplanned interruption of radiotherapy in breast cancer patients
}

\author{
Shiho Lee ${ }^{1} \cdot$ Jaesung Heo ${ }^{1}$ (D) \\ Received: 9 August 2021 / Accepted: 27 October 2021 / Published online: 5 November 2021 \\ (c) Springer Science+Business Media, LLC, part of Springer Nature 2021
}

\begin{abstract}
The purpose of this study was to investigate the impact of the coronavirus disease 2019 (COVID-19) pandemic on patients undergoing radiotherapy by comparing the patterns of unplanned radiotherapy interruption before and after the COVID-19 pandemic. We enrolled patients who received their first dose of radiotherapy for breast cancer between January 28 and July 31, 2019 and between January 28, 2020, and July 31, 2020. We compared the radiotherapy interruption patterns in 2019 with those in 2020 to analyze the impact of the COVID-19 pandemic on treatment interruption. Between January 28 and July 31, 2019, 287 patients with breast cancer received radiotherapy. Among them, 19 patients (6.6\%) experienced treatment interruption; the reasons for treatment interruption were radiotherapy-related side effects (10 patients, 52.6\%), other medical reasons (three patients, 15.8\%), and personal reasons (six patients, 31.6\%). Between January 28 and July 31, 2020, 279 patients with breast cancer received radiotherapy. Among them, 23 patients (8.2\%) experienced treatment interruption; the reasons for treatment interruption were radiotherapy-related side effects (eight patients, 35\%) and COVID-19 screening clinic-related reasons (six patients, 26.1\%). Among the six patients with screening clinic-related causes of radiotherapy interruption, five had asymptomatic fever and one had mild cold-like symptoms. The duration of treatment interruption was longer in patients with screening clinic-related interruptions than in those with interruptions because of other causes $(p=0.019)$. Multivariate analysis showed that cancer stage and radiotherapy volume did not significantly affect treatment interruption. The radiotherapy of certain patients was suspended despite the lack of a confirmed COVID-19 diagnosis. Precise and systematic criteria for the management of patients with suspected COVID-19 are needed, and the opinion of radiation oncologist in charge of the patient must also be considered.
\end{abstract}

Keywords COVID-19 $\cdot$ Breast cancer $\cdot$ Radiation therapy $\cdot$ Treatment interruption $\cdot$ Screening clinic

\section{Introduction}

In December 2019, an outbreak of a coronavirus variant was confirmed in Wuhan, China [1]. This virus subsequently spread worldwide. In March 2020, the World Health Organization declared the coronavirus disease 2019 (COVID-19) outbreak a global pandemic and it is still ongoing [2]. The first case of COVID-19 in Korea was reported in January 2020, with over 60,000 cases being reported since then [3]. At that time, the Korean Ministry of Health and Welfare

Jaesung Heo

md.js.informatics@gmail.com

1 Department of Radiation Oncology, Ajou University School of Medicine, 164 Worldcup-ro, Yeongtong-gu, Suwon 16499, Republic of Korea developed and distributed COVID-19 response guidelines [4].

Accordingly, our hospital set up and operated a screening clinic at its entrance from January 28, 2020. At this screening clinic, individuals visiting the hospital had their body temperature measured and were interviewed regarding respiratory symptoms, travel history, and contact history [5]. Patients with suspected COVID-19 were identified and restricted from entering the hospital. Since patients with cancer are at high risk of SARS-CoV-2 infection, caution is required, especially given the prolonged nature of the pandemic [6].

Most patients with cancer received radiotherapy 5 days a week on an outpatient basis [7]. Therefore, they had to undergo screening at the clinic before entering the hospital for each session. We especially selected patients with breast 
cancer because they accounted for the highest proportion of patients who received radiotherapy at our hospital.

During the course of screening, we noticed that patients who did not have COVID-19 were being suspected of having it and their treatment was being suspended for several days. Consequently, we aimed to qualify the actual state of radiotherapy interruption caused by the establishment of a COVID-19 screening clinic and to analyze the factors affecting it. Ultimately, in this study, we intend to provide a basis to establish guidelines for the management of patients with cancer who require radiotherapy from the hospital during the COVID-19 pandemic.

\section{Methods}

Patients with breast cancer who were receiving radiotherapy at our hospital were included in this study. Breast cancer is treated with surgery, chemotherapy, and radiotherapy. However, these treatments are performed sequentially, not simultaneously. Therefore, the effect of COVID-19 screening on the interruption of each type of treatment can be individually analyzed. If the patients scheduled to receive radiotherapy were suspected of having COVID-19, radiotherapy was not administered [8]. Instead, they underwent COVID-19 testing. If they tested positive, radiotherapy was resumed only after complete recovery from COVID-19. On the other hand, if they tested negative, radiotherapy was resumed immediately.

All patients in this study received their first dose of radiotherapy between January 28, 2020, when the screening clinic at our hospital was established, and July 31, 2020. To determine the differences in radiotherapy interruption before and after the screening clinic was established, we included a comparison group of patients with breast cancer who received their first dose of radiotherapy between January 28, 2019 and July 31, 2019. This study was approved by the institutional review board of Ajou University Hospital (IRB No. AJIRB-MED-MDB-21-027). The requirement for informed consent was waived by the board. All methods were performed in accordance with the Declaration of Helsinki.

Data regarding clinical factors such as age, cancer stage, chemotherapy history, and radiotherapy volume were collected from our hospital's electronic medical record system. Cancer stage was based on the pathologic stage in patients who did not receive neoadjuvant chemotherapy and the clinical stage before chemotherapy in patients who received neoadjuvant chemotherapy. Radiotherapy volume was classified into irradiation of only one breast (breast only) and irradiation of one breast along with other areas (extended field). An extended field included one or more lower axillary structures, the internal mammary lymph nodes, the supraclavicular lymph nodes, and the contralateral breast.

Data regarding radiotherapy interruption were also collected from electronic medical records. If a patient did not receive radiotherapy according to the initially set treatment schedule, the duration and cause of treatment interruption were noted. The causes of treatment interruption were categorized as radiotherapy-related side effects, personal reasons, other medical reasons, and screening-related interruptions. Between-group differences in covariate distribution were determined using the chi-square test for categorical variables and t test for continuous variables. A two-sided $p$-value of $<0.05$ was considered statistically significant. Odds ratios with $95 \%$ confidence intervals were obtained on multivariate logistic regression analysis to estimate the relative risk of radiotherapy interruption associated with each clinical factor. All statistical analyses were performed using R studio version 3.6.3 (R foundation for Statistical Computing, Vienna, Austria).

\section{Results}

A total of 287 and 279 patients with breast cancer received radiotherapy between January 28 and July 31, 2019 and between January 28 and July 31, 2020, respectively. Among them, unplanned radiotherapy interruption occurred in 19 of $287(6.6 \%)$ patients in 2019 and in 23 of $279(8.2 \%)$ patients in 2020 .

The causes of radiotherapy interruption in 2019 were radiotherapy-related side effects (10 patients, 53\%), personal reasons, such as vacation and family problems (six patients, $31 \%$ ), and other medical reasons, such as exacerbation of underlying medical conditions and traffic accidents (three patients, $16 \%$ ), whereas those in 2020 were radiotherapyrelated side effects (eight patients, 35\%), personal reasons (five patients, 22\%), other medical reasons (four patients, $17 \%$ ), and screening clinic-related interruptions (six patients, $26 \%$; Fig. 1). Among the six patients who were denied entry to the hospital at the screening clinic, five had asymptomatic fever and one had cold-like respiratory symptoms. These six patients underwent COVID-19 testing, and all of them tested negative.

Table 1 compares the characteristics of patients whose radiotherapy was interrupted in 2019 and 2020 . There were no significant between-group differences in age, duration of treatment interruption, cancer stage, and radiotherapy volume. We analyzed the association between clinical factors for cases in which radiotherapy was not interrupted and for cases in which it was interrupted (Table 2), in 2020 when the COVID-19 pandemic spread. No clinical factors of significance were identified. Table 3 compares the characteristics of patients with screening clinic-related 


\section{BREAST CANCER PATIENT INTERRUPTION CAUSE $19 / 287$ (6.6\%)}

\section{BREAST CANCER PATIENT INTERRUPTION CAUSE $23 / 279(8.2 \%)$}
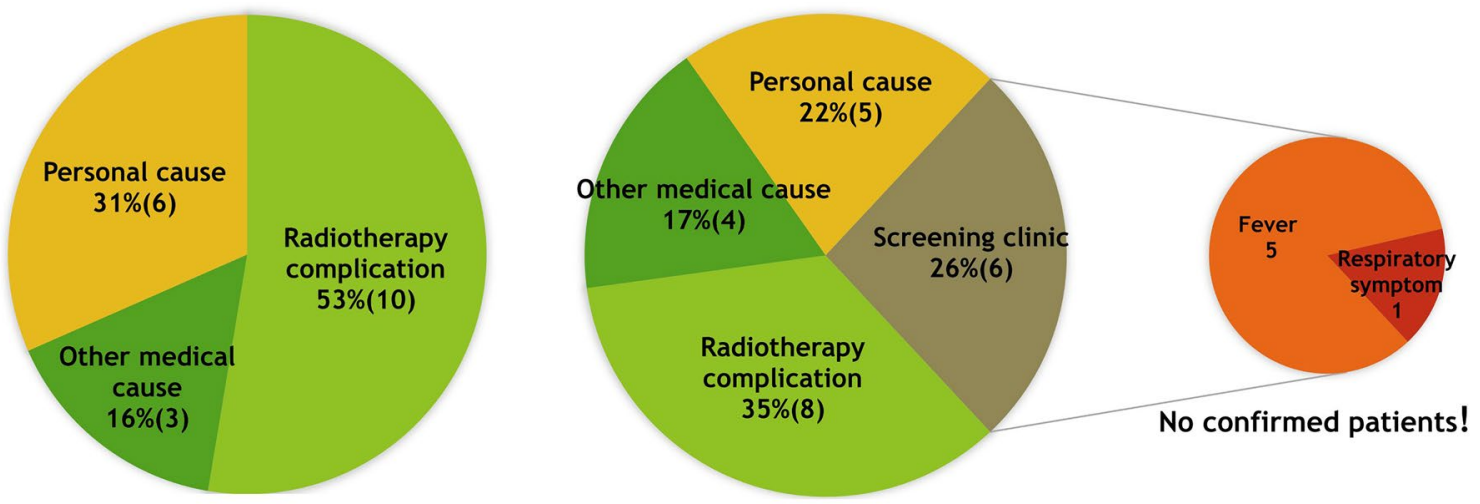

Fig. 1 Comparison of the causes of radiotherapy interruption in 2019 and 2020

Table 1 Comparison of the clinical features of patients with radiotherapy interruption in 2019 and 2020

\begin{tabular}{lcc}
\hline & $2019(n=19)$ & $2020(n=23)$ \\
\hline Age & $55.0(47.0-59.5)$ & $49.0(44.5-57.5)$ \\
Interruption period (day) & $1.0(1.0-2.5)$ & $2.0(1.0-5.0)$ \\
Stage & & \\
0 & $4(21.1 \%)$ & $2(8.7 \%)$ \\
I & $7(36.8 \%)$ & $9(39.1 \%)$ \\
II & $8(42.1 \%)$ & $8(34.8 \%)$ \\
III & $0(0 \%)$ & $3(13.0 \%)$ \\
IV & $0(0 \%)$ & $1(4.3 \%)$ \\
Chemotherapy history & & \\
No & $13(68.4 \%)$ & $8(34.8 \%)$ \\
Yes & $6(31.6 \%)$ & $15(65.2 \%)$ \\
Radiation volume & & $13(56.5 \%)$ \\
Breast only & $14(73.7 \%)$ & $10(43.5 \%)$ \\
Extended field & $5(26.3 \%)$ & \\
\hline
\end{tabular}

radiotherapy interruptions and radiotherapy interruptions of other causes in 2020.

Patients with screening clinic-related interruptions had a significantly longer duration of treatment interruption than those with interruptions because of other causes $(p=0.019)$. These patients also tended to have a higher cancer stage and larger radiotherapy volume than those with interruptions of other causes, but this was not statistically significant. Table 4 shows the results of the multivariable analysis of the effects of cancer stage and radiotherapy volume on treatment interruption. Neither variable showed a statistically significant effect.

\section{Discussion}

Unplanned radiotherapy interruption occurred in $6.6 \%$ and $8.2 \%$ of patients in 2019 and 2020 , respectively, with a $24 \%$ increase in the proportion of patients with radiotherapy interruptions from 2019 to 2020. Screening clinic-related interruptions accounted for $26 \%$ of all radiotherapy interruptions in 2020, and when these were removed from the analysis, there was no significant difference in the rates of radiotherapy interruptions between 2019 and 2020 (6.6\% and $6.1 \%$, respectively). This study confirmed that COVID19 screening was a new cause of radiotherapy interruption in 2020.

All patients visiting the hospital, including those who required radiotherapy, were administered a basic questionnaire for COVID-19 (respiratory symptoms, history of travel to high-risk areas, history of contact with suspected COVID19 patients) and underwent body temperature measurements. For South Korea, fortunately, medical resources were available and dedicated medical personnel, including doctors and nurses, were assigned to take charge of the screening. Patients with unusual findings were referred to a physician at the screening clinic. The physician was not the patients' primary care physician, but an internal or emergency medicine specialist assigned to the screening area by the hospital. This physician, per their clinical judgment, decided whether or not to conduct a COVID-19 test and whether patients could enter the hospital premise.

Routine pre-radiotherapy COVID-19 screening was not performed because basic medical examinations and body temperature measurements were performed at the screening clinic of the hospital. However, if fever or respiratory symptoms occurred during treatment, the decision to continue treatment was made at the discretion of the radiation 
Table 2 Comparison between the clinical features of patients with and without radiotherapy interruption in 2020
Table 3 Comparison of the clinical features of patients with screening-related radiotherapy interruption and those with radiotherapy interruption because of other causes in 2020

\begin{tabular}{lccc}
\hline & No interruption $(n=256)$ & Interruption $(n=23)$ & $p$-value \\
\hline Age & $51.0(46.0-59.0)$ & $49.0(44.5-57.5)$ & 0.452 \\
Stage & & & \\
0 & $25(9.8 \%)$ & $2(8.7 \%)$ & 0.278 \\
I & $115(44.9 \%)$ & $9(39.1 \%)$ & \\
II & $91(35.5 \%)$ & $8(34.8 \%)$ & \\
III & $24(9.4 \%)$ & $3(13.0 \%)$ & 0.494 \\
IV & $1(0.4 \%)$ & $1(4.3 \%)$ & \\
Chemotherapy history & & $8(34.8 \%)$ & \\
No & $114(44.5 \%)$ & $15(65.2 \%)$ & 0.274 \\
Yes & $152(55.5 \%)$ & $13(56.5 \%)$ & \\
Radiation volume & $179(69.9 \%)$ & $10(43.5 \%)$ & \\
Breast only & $77(30.1 \%)$ & & \\
Extended field & & & \\
\hline
\end{tabular}

\begin{tabular}{lccc}
\hline & $\begin{array}{l}\text { Non-screening-related inter- } \\
\text { ruption }(n=17)\end{array}$ & $\begin{array}{l}\text { Screening-related interrup- } \\
\text { tion }(n=6)\end{array}$ & $p$-value \\
\hline Age & $49.4(45.0-58.0)$ & $50.5(44.0-57.0)$ & 0.944 \\
Interruption period (day) & $1.0(1.0-2.0)$ & $5.0(3.0-6.0)$ & 0.019 \\
Stage & & & \\
0 & $2(11.8 \%)$ & $0(0 \%)$ & \\
I & $7(41.2 \%)$ & $2(33.3 \%)$ & \\
II & $7(41.2 \%)$ & $1(16.7 \%)$ & \\
III & $1(5.9 \%)$ & $2(33.3 \%)$ & \\
IV & $0(0 \%)$ & $1(16.7 \%)$ & \\
Chemotherapy history & & $1(16.7 \%)$ & \\
No & $7(41.2 \%)$ & $5(83.3 \%)$ & \\
Yes & $10(58.8 \%)$ & $1(16.7 \%)$ & \\
Radiation volume & & $5(83.3 \%)$ & \\
Breast only & $5(70.6 \%)$ & & \\
Extended field & $5(29.4 \%)$ & & \\
\hline
\end{tabular}

Table 4 Results of the multivariable analysis of the factors affecting radiotherapy interruption

\begin{tabular}{lll}
\hline & OR $(95 \% \mathrm{CI})$ & $p$ value \\
\hline Stage & $1.63(0.38-7.06)$ & 0.515 \\
Radiotherapy volume & $6.65(0.34-124.51)$ & 0.211 \\
\hline
\end{tabular}

oncologist in charge. There were no cases of delayed treatment unless an actual diagnosis of COVID-19 was confirmed or the patient's general condition was particularly poor.

As seen in Table 2, the duration of treatment interruption is significantly longer in patients with screening clinicrelated interruption than in those with other causes of interruption. This is due to the protocol followed for patients with suspected COVID-19. Five patients with asymptomatic fever were instructed to rest for a day and a COVID-19 test was not immediately conducted because these patients had no significant travel or contact history. If the fever persisted, COVID-19 testing was performed. Furthermore, these patients were denied entry to the hospital despite testing negative if they were febrile. Since most of the decisions regarding hospital admission were made by the doctors at the screening clinics, the opinions of the radiation oncologists in charge of the patients were not considered in some cases, resulting in interruption of treatment for longer than necessary.

We discontinued treatment if COVID-19 was suspected. This was due to the structure of the radiation oncology department at our hospital; it was impossible to separate the areas (waiting room, changing room, toilet, treatment room) for the patients with suspected COVID-19 and those not suspected of it. Due to the high infectivity rate of COVID-19 
and the relatively low immunity in patients with cancer receiving radiotherapy, treatment was temporarily suspended for patients with suspected COVID-19 to prevent a cluster infection that could pose a serious threat.

Since most of the suspected patients often turned out to be COVID-19 negative, our department requested the screening clinic to actively test for COVID-19 in patients with fever receiving radiotherapy. Patients who tested negative for COVID-19 requested that they must be allowed to enter the hospital even if they had a fever. To proceed safely with patients who resumed treatment after COVID-19 recovery, our department conducted additional procedures to measure body temperatures and check respiratory symptoms. Through these strict standards, there was no case of cluster infection at our radiation oncology department, despite the high risk of infection.

Although multivariate analysis did not yield statistically significant results, patients with screening clinic-related interruptions tended to have a higher cancer stage and larger radiotherapy volume than those with interruptions of other causes. Five out of the six patients with screening clinicrelated interruptions were denied entry into the hospital due to asymptomatic fever, which we believe was related to their cancer and not to COVID-19. The most common causes of fever in patients with cancer are infection, cancerrelated fever, and chemotherapy or radiotherapy $[9,10]$. Increased frequency of occurrence of cancer-related fever has been associated with a high cancer stage and large radiotherapy volume $[11,12]$. None of the patients with screening clinic-related interruptions were actually confirmed to have COVID-19. Therefore, their fever was probably not caused by COVID-19, but was due to other causes, leading to unnecessary interruption of radiotherapy. In such cases, radiotherapy termination may affect its effectiveness, and it is important to quickly identify the cause of the fever, following which the duration of treatment interruption should be minimized [13].

Although it is debated, the therapeutic effect of radiotherapy may decrease when the same dose is administered after a long gap in treatment [14]. The additional radiotherapy has been recommended in cases of treatment interruption beyond a certain duration [15]. However, this was not done at our hospital, and our patients with breast cancer received their treatment at the originally planned radiation dose. In the case of head and neck cancer, discontinuation of treatment negatively affects the prognosis [16]. For breast cancer, a study reported that discontinuation of treatment for more than one week affects prognosis [17]. However, in our case, the duration of treatment discontinuation was less than one week. This was because there were no confirmed COVID-19 cases and in most cases, treatment resumed as soon as the fever subsided. However, if the duration of treatment discontinuation is more than one week, additional treatment may need to be considered to compensate for the loss in treatment time. Therefore, additional research is needed on whether a reduced treatment effect is observed in patients with COVID-19 screening clinic-related radiotherapy interruption.

An important finding of this study is that there were patients at our hospital whose radiotherapy was suspended for longer than necessary even though they did not have COVID-19. Although screening clinics are effective in preventing patients with COVID-19 from entering hospitals, they also prevent patients with cancer from receiving adequate treatment. To solve this problem, precise and systematic criteria for the classification of patients with suspected COVID-19 are needed, and the opinion of the radiation oncologist in charge of the patient must also be considered. This study makes a novel contribution that it has the potential to inspire future studies and guide policy making regarding the screening of patients with cancer aimed at minimizing unnecessary treatment interruptions.

The single-center nature and short duration are the limitations of this study. Since the study was conducted at only one institution over a period of approximately 6 months, the number of patients studied was small, and there were only six patients with screening clinic-related treatment interruption. In addition, we only included patients from 2019 in the control group, leading to uncertain reliability. Further long-term multicenter studies are needed to analyze the effect of COVID-19 screening clinics on interruption of radiotherapy.

In conclusion, the COVID-19 pandemic has been ongoing for a long time, resulting in changes in the treatment environment of patients with cancer. We found that the establishment of a COVID-19 screening clinic became a barrier to hospital access and a new major cause of interruption of radiotherapy in patients with breast cancer. We also found that COVID-19 screening led to radiotherapy interruption even in patients who did not have COVID-19. This should be considered when formulating guidelines for screening patients receiving radiotherapy.

Acknowledgements We would like to thank Editage (www.editage. co.kr) for editing and reviewing this manuscript for English language.

Funding This research was supported by the Bio and Medical Technology Development Program of the National Research Foundation (NRF) funded by the Korean government (MSIT) (No. 2020M3A9E8024907). This research was supported by a Grant of the Korea Health Technology R\&D Project through the Korea Health Industry Development Institute (KHIDI), funded by the Ministry of Health and Welfare, Republic of Korea (Grant number: HR21C1003).

\section{Declarations}

Conflict of interest The authors declare that they have no conflict of interest. 


\section{References}

1. Wu Z, McGoogan JM. Characteristics of and important lessons from the coronavirus disease 2019 (COVID-19) outbreak in china: summary of a report of 72314 cases from the Chinese center for disease control and prevention. JAMA. 2020;323:1239-42. https://doi.org/10.1001/jama.2020.2648.

2. Cucinotta D, Vanelli M. WHO declares COVID-19 a pandemic. Acta Biomed. 2020;91:157-60. https://doi.org/10.23750/abm. v91i1.9397.

3. Dighe A, Cattarino L, Cuomo-Dannenburg G, Skarp J, Imai N, Bhatia S, Gaythorpe KAM, Ainslie KEC, Baguelin M, Bhatt S, et al. Response to COVID-19 in South Korea and implications for lifting stringent interventions. BMC Med. 2020;18:321. https:// doi.org/10.1186/s12916-020-01791-8.

4. Kang J, Jang YY, Kim J, Han S-H, Lee KR, Kim M, Eom JS. South Korea's responses to stop the COVID-19 pandemic. Am J Infect Control. 2020;48:1080-6. https://doi.org/10.1016/j.ajic. 2020.06.003.

5. Kim J-H, An JA-R, Min P-K, Bitton A, Gawande AA. How South Korea responded to the Covid-19 outbreak in Daegu. NEJM Catal. 2020. https://doi.org/10.1056/CAT.20.0159.

6. Jazieh AR, Akbulut H, Curigliano G, Rogado A, Alsharm AA, Razis ED, Mula-Hussain L, Errihani H, Khattak A, Guzman RBD, et al. Impact of the COVID-19 pandemic on cancer care: a global collaborative study. JCO Glob Oncol. 2020. https://doi.org/10. 1200/go.20.00351.

7. Haussmann J, Corradini S, Nestle-Kraemling C, Bölke E, Njanang FJD, Tamaskovics B, Orth K, Ruckhaeberle E, Fehm $\mathrm{T}$, Mohrmann S, et al. Recent advances in radiotherapy of breast cancer. Radiat Oncol. 2020;15:71. https://doi.org/10.1186/ s13014-020-01501-X.

8. Lee JB, Jung M, Kim JH, Kim BH, Kim Y, Kim YS, Kim BC, Kim J, Moon SH, Park K-U, et al. Guidelines for cancer care during the COVID-19 pandemic in South Korea. Cancer Res Treat. 2021;53:323-9. https://doi.org/10.4143/crt.2020.1256.

9. Ogawara D, Fukuda M, Ueno S, Ohue Y, Takemoto S, Mizoguchi K, Nakatomi K, Nakamura Y, Obase Y, Honda T, et al. Drug fever after cancer chemotherapy is most commonly observed on posttreatment days 3 and 4. Support Care Cancer. 2016;24:615-9. https://doi.org/10.1007/s00520-015-2820-8.

10. Morabito F, Irrera G, Oliva E, Console G, Martino M, Pucci G, Messina G, Barbaro P, Palazzo S, Iacopino P. Infectious complications in breast cancer patients undergoing peripheral blood stem cell transplantation: a single center retrospective analysis towards outpatient strategy. Bone Marrow Transplant. 2001;28:883-8. https://doi.org/10.1038/sj.bmt.1703246.

11. Benveniste MF, Gomez D, Carter BW, Cuellar SLB, Shroff GS, Benveniste APA, Odisio EG, Marom EM. Recognizing radiation therapy-related complications in the chest. Radiographics. 2019;39:344-66. https://doi.org/10.1148/rg.2019180061.

12. Foggo V, Cavenagh J. Malignant causes of fever of unknown origin. Clin Med (London). 2015;15:292-4. https://doi.org/10.7861/ clinmedicine.15-3-292.

13. Yu D, Hu W, Chen L, Fu Z, Song Q, Li X. Effect of radiotherapy interruption due to COVID-19 outbreak. Radiother Oncol. 2021;155:1-2. https://doi.org/10.1016/j.radonc.2020.09.055.

14. González Ferreira JA, Jaén Olasolo J, Azinovic I, Jeremic B. Effect of radiotherapy delay in overall treatment time on local control and survival in head and neck cancer: review of the literature. Rep Pract Oncol Radiother. 2015;20:328-39. https://doi.org/ 10.1016/j.rpor.2015.05.010.

15. Chen YP, Tsang NM, Tseng CK, Lin SY. Causes of interruption of radiotherapy in nasopharyngeal carcinoma patients in Taiwan. Jpn J Clin Oncol. 2000;30:230-4. https://doi.org/10.1093/jjco/hyd059.

16. Nagar H, Formenti SC. Cancer and COVID-19 - potentially deleterious effects of delaying radiotherapy. Nat Rev Clin Oncol. 2020;17:332-4. https://doi.org/10.1038/s41571-020-0375-1.

17. Bese NS, Sut PA, Ober A. The effect of treatment interruptions in the postoperative irradiation of breast cancer. Oncology. 2005;69:214-23. https://doi.org/10.1159/000087909.

Publisher's Note Springer Nature remains neutral with regard to jurisdictional claims in published maps and institutional affiliations. 